\title{
A Case of Hepatitis B Infection; Is It Transmitted via Blood Components?
}

\author{
Sedigheh Amini Kafiabad ${ }^{1}$; Hayedeh Javadzadeh Shahshahani ${ }^{1, *}$; Mahtab Vaziri ${ }^{1}$ \\ ${ }^{1}$ Blood Transfusion Research Center, High Institute for Research and Education in Transfusion Medicine, Tehran, IR Iran \\ ${ }^{*}$ Corresponding author: Hayedeh Javadzadeh Shahshahani, Blood Transfusion Research Center, High Institute for Research and Education in Transfusion Medicine, Tehran, IR Iran. \\ Tel:+98-3518243300, E-mail: javadzadehhayedeh@gmail.com
}

Received: October 9, 2014; Revised: December 20, 2014; Accepted: December 29, 2014

\begin{abstract}
Introduction: Hepatitis B infection remains a major health problem worldwide and is an important cause of morbidity and mortality. Since it is transmitted parenterally, investigation of new cases of Hepatitis B infections for potential routes of transmission plays a crucial role in controlling hepatitis B infection in the community.

Case Presentation: In this study, we reported a new case of hepatitis B infection. Although the case had been exposed to different routes of transmission, only transmission via blood transfusion was evaluated.

Conclusions: To improve infection control in the community, strategies should be performed to investigate more frequent routes of transmission and not only transfusion.
\end{abstract}

Keywords:Hepatitis B; Transmission; Blood Transfusion; Health-Care

\section{Introduction}

Hepatitis B infection is caused by a DNA virus and may remain indolent or result in hepatic inflammation and fibrosis. Hepatitis B Virus (HBV) has infected more than two billion people, approximately one-third of the world's population. HBV infection is an important cause of morbidity and mortality in the world. Viral hepatitis B infection is transmitted parenterally, usually in the perinatal period or sexual contact or intravenous drug use. Acupuncture, tattooing and haemodialysis are other infrequent routs of transmission (1-3). The risk of transfusion transmitted (TT) HBV infection has been continuously decreased since the introduction of hepatitis B surface antigen (HbsAg) testing in the early 1970's, but HBV remains a considerable risk for TT infection (4). Since $\mathrm{HBV}$ is transmitted efficiently percutaneously, possible transmission in health-care settings is also of particular concern (5). Therefore, public health investigations of cases of HBV infection for possible routes of transmission play an essential role in controlling viral hepatitis in community.

\section{Case Presentation}

In September 2012, a 56-year-old man referred to Yazd blood transfusion center for evaluation of possibility of post-transfusion hepatitis B infection. In his medical history, he had undergone an axillobifemoral bypass surgery in February 2012 without receiving any blood products. He had also undergone angiography and stent implantation because of extensive myocardial infarction in August 2011. At that time, the results of laboratory tests for HBs-Ag, HCV-Ab and HIV-Ab were negative. In May 2012, he was hospitalized due to hematochezia and epistaxis because of warfarin overdose. He received one unit of fresh frozen plasma (FFP) and three units of RBCs because of prolonged Prothrombin Time (PT), Partial Thromboplastin Time (PTT) and anaemia. In July 2012, he was referred to hospital with fever, jaundice and purulent discharge from surgical scar. His serum sample had positive results for HBs-Ag, HBe-Ag and Anti-HBc IgM. Serum levels of bilirubin, Alanine transaminase (ALT) and Aspartate aminotransferase (AST) increased by twentyfold (Table 1). The diagnosis was hepatitis B infection. He received two units of FFP and intravenous antibiotics. He was discharged after 19 days, while liver enzyme tests levels were decreased and his general condition was good. In Yazd blood transfusion center, a consultant physician interviewed the case for other risk factors. The case had been imprisoned during 1997-99. He had other highrisk behaviors such as homosexuality, tattooing, being injured by knife in street fights and addiction to opioid drugs in his history. Trace back was performed in Yazd blood center. Donors who donated blood products to the recipient were recalled using data registry software to collect a blood sample to test HBV markers. In addition, all plasma products donated previously by these donors not still used were tested. All samples had negative results for HBsAg (Monolisa HBsAg Ultra, Bio-Rad, Marnes La Coquette, France), Anti-HBc Ab (Enzygnost, Siemens, Marburg, Germany) and HBV DNA by Real ART HBV LC PCR kit (Artus GmbH, Hamburg, Germany) (Table 2).

Copyright (C) 2015, Infectious Diseases and Tropical Medicine Research Center. This is an open-access article distributed under the terms of the Creative Commons Attribution-NonCommercial 4.0 International License (http://creativecommons.org/licenses/by-nc/4.0/) which permits copy and redistribute the material just in noncommercial usages, provided the original work is properly cited. 
Table 1. Laboratory Test Results of Patient in the Last Hospitalization $^{\mathrm{a}}$

\begin{tabular}{lc}
\hline Laboratory Test & Results \\
\hline Hemoglobin, g/L & 131 \\
PT, $\mathbf{S}$ & 33 \\
\hline PTT, $\mathbf{S}$ & 45 \\
\hline INR & 6.4 \\
\hline Bilirubin total, mg/dL & 14.8 \\
\hline Bilirubin direct, $\mathbf{m g / d L}$ & 7.6 \\
\hline ALT, IU/L & 804 \\
\hline AST, IU/L & 1350 \\
\hline ALP, IU/L & 1161 \\
\hline HBs-Ag & Positive \\
\hline HBe-Ag (ELFA) & Positive \\
Anti-HBc(IgM) & Positive \\
\hline
\end{tabular}

a Abbreviations: Alkaline phosphatase: ALP; International Normalized Ratio: INR

\section{Discussion}

The risk of transfusion-transmitted HBV infection today is lower than ever by continuous improvement and implementation of donor selection and sensitive screening tests; however, blood born infections may occur if donors donate blood in the window period or in the phases of infection with very low viremia (4). Implementation of look back program to trace the products collected from a donor to the final recipients and from the recipient back to the donor, preferably by means of a computer database, has increased blood supply safety.

This procedure is followed when it is determined retrospectively that a blood or plasma donation should have been excluded from processing for instance, because the unit was collected from a donor who was subsequently rejected for reactive viral marker, high-risk behavior or other risks related to infectious diseases (donor lookback). There is a process for investigating a report of a suspected transfusion-associated reaction in a recipient, to identify a potentially implicated donor (recipient lookback). The donor of products implicated in transmitting disease or causing recipient harm is excluded from fur- ther donations. All other donations from the implicated donor are traced and blood components removed from the inventory and recalled, if within the expiry date. All post-donation information are recorded and maintained. There is a system in place to react accordingly and in time to remove unexpired products from distribution to assure the safety of recipients.

In Iran, hepatitis B surface antigen (HBsAg) in serum is the seromarker to indicate HBV infection in blood donors and it is routinely included in the donor screening. Iran with a $3 \%$ prevalence of HBsAg was previously an intermediate endemic country for HBV infection. Since the adoption of routine HBV neonatal vaccination from 1993, it is estimated that HBV endemicity has decreased reaching to $1.7 \%$ (6). Risk of transmission of HBV through HBs-Ag negative blood products is one per 63000-100000 donations (7). For the presented case, all documentations in blood transfusion center for blood donors and patient documentations in hospitals were evaluated for transmission of HBV via blood components and HBV markers of blood donations had negative results (Table 2). Therefore, transmission of Hepatitis B via blood transfusion is unlikely. There are many reports of tracing blood units from recipients to their donors. In a recent study, Allain and his coworkers investigated recipients of previous donations through look back or trace back. They concluded that blood components from donors with occult hepatitis B carried a high risk of HBV transmission by transfusion (8). In a similar study in Iran, tracing back of blood donations showed no transfusion transmitted HBV (7).

For the case studied, other much more prevalent routes of transmission such as high-risk behaviors or healthcare-associated transmission should be considered. This case had many high-risk behaviors in his past and perhaps he had been a case of occult HBV infection reactivated during his recent medical condition. There are several studies showing reactivation of HBV during immunosuppression or some other medical conditions. In a recent study, a patient with positive results for anti-HBs and anti-HBc presented HBV reactivation in a context of ischemic stroke, with no other intercurrent iatrogenic phenomenon or usual immunosuppressive pathology (9). However, in the present study, the case had no history of anti-HBc, anti-HBs or HBV DNA testing in the past to

\begin{tabular}{lcccccccc}
\hline \multicolumn{7}{l}{ Table 2. Laboratory Test Results of Donors Who Donated Blood Components to the Case } \\
\hline Donor & Gender & Marital Status & Age, $\mathbf{y}$ & Donation Status & HBs-Ag & Anti-HBc & Anti-HBs, IU/L & HBV DNA PCR \\
\hline $\mathbf{1}$ & Male & married & 23 & Regular & Negative & Negative & $>100$ & Negative \\
$\mathbf{2}$ & Male & married & 63 & Regular & Negative & Negative & Negative & Negative \\
$\mathbf{3}$ & Male & married & 34 & Lapsed & Negative & Negative & Negative & Negative \\
$\mathbf{4}$ & Male & married & 44 & Regular & Negative & Negative & Negative & Negative \\
$\mathbf{5}$ & Male & married & 23 & Regular & Negative & Negative & $>100$ & Negative \\
$\mathbf{6}$ & Male & married & 25 & Regular & Negative & Negative & Negative & Negative \\
\hline
\end{tabular}


confirm this supposition. Since transfusion-transmitted infections are often a cause for legal procedures, collection of a pre-transfusion recipient sample kept as archive would be critical in determining the transfusion origin of an HBV infection (5). On the other hand, the presented case received health care in medical centers prior to diagnosis of HBV infection. The possibility of health-care associated transmission was not evaluated for the case. Transmission of HBV in health-care settings is an increasingly recognized public health problem (4). Public health investigations of cases of $\mathrm{HBV}$ infection suspected to be associated with health-care delivery play an essential role in identifying unsafe practices and controlling healthcare-associated viral hepatitis transmission. However, these investigations are resource intensive and pose numerous challenges. In a recent study, a systematic approach was reported to guide investigation and public health response to case reports of acute $\mathrm{HBV}$ or $\mathrm{HCV}$ infection in patients whose infection was potentially associated with health-care delivery that might be useful to develop this type of investigation (5).

In conclusion, strategies should be performed to investigate frequent routes of transmission to ensure patients safety and improve infection control in the community.

\section{Authors' Contributions}

H. Javadzadeh Shahshahani wrote the introduction and discussion. M. Vaziri collected data and wrote the case history and S. Amini reviewed the manuscript.

\section{References}

1. Alavian SM, Behnava B. What is the Reason for Poor Outcome of Antepartum Immunoprophylaxis of Hepatitis B Immunoglobulin in Prevention of Vertical Hepatitis B Transmission? Hepat Mon. 2007;7(3):163-5.

2. Franco E, Bagnato B, Marino MG, Meleleo C, Serino L, Zaratti L. Hepatitis B: Epidemiology and prevention in developing countries. World J Hepatol. 2012;4(3):74-80.

3. Lok AS, McMahon BJ. Chronic hepatitis B: update 2009. Hepatology. 2009;50(3):661-2.

4. Bihl F, Castelli D, Marincola F, Dodd RY, Brander C. Transfusiontransmitted infections. J Transl Med. 2007;5:25.

5. Centers for Disease C, Prevention.. Investigation of viral hepatitis infections possibly associated with health-care delivery-New York City, 2008-2011. MMWR Morb Mortal Wkly Rep. 2012;61(19):3338.

6. Vaezjalali M, Rashidpour S, Rezaee H, Hajibeigi B, Zeidi M, Gachkar L, et al. Hepatitis B viral DNA among HBs antigen negative healthy blood donors. Hepat Mon. 2013;13(3).

7. Amini S, Talebian A, Pourfath EA. Tracing Blood Units From Recipients To Their Donors: Results Of A Trace Back Study For Hepatitis B. Blood. 2004;5:67-73.

8. Allain JP, Mihaljevic I, Gonzalez-Fraile MI, Gubbe K, Holm-Harritshoj L, Garcia JM, et al. Infectivity of blood products from donors with occult hepatitis B virus infection. Transfusion. 2013;53(7):1405-15.

9. Deroux A, Larrat S, Hilleret MN, Baccard M, Qin W, Billet G, et al. Case report: detection of a hepatitis $B$ surface antigen variant emerging in an elderly patient after an ischemic cerebral vascular accident. J Med Virol. 2012;84(12):1897-900. 